\title{
Time-Resolved Small-Angle X-Ray Scattering Reveals Millisecond Transitions of a DNA Origami Switch
}

\author{
Linda K. Bruetzel ${ }^{1, \dagger}$, Philipp U. Walker ${ }^{1, \dagger}$, Thomas Gerling ${ }^{2}$, Hendrik Dietz ${ }^{2}$, Jan Lipfert ${ }^{1, *}$ \\ ${ }^{1}$ Department of Physics, Nanosystems Initiative Munich, and Center for Nanoscience, LMU \\ Munich, Amalienstrasse 54, 80799 Munich, Germany \\ ${ }^{2}$ Physik Department, Walter Schottky Institute, Technische Universität München, Am \\ Coulombwall 4a, 85748 Garching near Munich, Germany \\ ${ }^{\dagger}$ These authors contributed equally to this work. \\ *Corresponding author: E-mail: Jan.Lipfert@1mu.de. Phone: +49-89-2180-2005
}

\begin{abstract}
Self-assembled DNA structures enable creation of specific shapes at the $\mathrm{nm}-\mu \mathrm{m}$ scale with molecular resolution. The creation of functional DNA assemblies will likely require dynamic structures that can undergo controllable conformational changes. DNA devices based on shape complementary stacking interactions have been demonstrated to undergo reversible conformational changes triggered by changes in ionic environment or temperature. An experimentally unexplored aspect is how quickly conformational transitions of large synthetic DNA origami structures can actually occur. Here, we use time-resolved small-angle X-ray scattering to monitor large-scale conformational transitions of a two-state DNA origami switch in free solution. We show that the DNA device switches from its open to its closed conformation upon addition of $\mathrm{MgCl}_{2}$ in milliseconds, which is close to the theoretical diffusive speed limit. In contrast, measurements on the dimerization of DNA origami bricks occur on a time scale of minutes to hours suggesting that the kinetics depend on local concentration and molecular alignment.
\end{abstract}

\section{KEYWORDS}

DNA nanotechnology, DNA origami, small angle X-ray scattering, SAXS, time-resolved SAXS, conformational changes 
The programmable self-assembly of DNA molecules is a new paradigm for creating structures

at the $\mathrm{nm}-\mu \mathrm{m}$ scale with potential for diagnostic, therapeutic, and engineering applications. ${ }^{1-4}$ DNA origami structures are assembled from kilobase long single-stranded (ss) DNA (scaffold) strands that fold due to a number of specific ssDNA oligonucleotides (staple strands) into pre-defined and precisely addressable $\sim$ MDa-sized structures. ${ }^{5-7}$ Recently, further hierarchical organization of DNA origami structures into higher-order assemblies, some of which reaching the $\sim$ GDa range, has been demonstrated ${ }^{8-10}$. Moving beyond static two- and three dimensional structures ${ }^{5,6}$ toward building complex devices will require implementing and controlling reversible mechanical movements in DNA objects, which remains a challenging aspect in the field. ${ }^{11}$ So far, most switchable DNA devices have been triggered by the addition of DNA single strands and toehold-mediated strand exchange, where transitions occur on time scales of minutes. Conformational changes controlled by the addition and exchange of oligonucleotides are fundamentally constrained by the maximal rate of toehold-mediated strand exchange $\sim 5 \cdot 10^{5} \mathrm{M}^{-1} \cdot \mathrm{s}^{-1}{ }^{12}$, at typical $\mathrm{nM}-\mu \mathrm{M}$ concentrations this corresponds to changes on the time scale of seconds to minutes ${ }^{13-16}$. Conformational transitions initiated by changes in ionic strength, $\mathrm{pH}$, temperature, or light can be faster, and have been demonstrated to occur within seconds. ${ }^{17,18}$ A new technology to create welldefined and reversible conformational changes of DNA origami devices relies on shape complementary protrusions and recessions that interact via DNA stacking interactions. ${ }^{19,20}$ While salt and temperature dependent conformational changes of DNA devices based on shape complementary and stacking interactions have been characterized, ${ }^{19,20}$ it is an open question how fast conformational transitions of these large, $\sim \mathrm{MDa}$ DNA origami structures can be. Friction with the solvent and energy barriers in junctions or pivots could pose fundamental speed limits for switching dynamics, similar to what is observed for folding of naturally occurring RNAs due to their rugged free energy landscapes (Supporting Fig. S1). ${ }^{21}$ 22

\section{Fast conformational transition of a DNA origami switch device revealed by trSAXS}

To address this question, we employ time-resolved small-angle X-ray scattering (trSAXS) to monitor conformational transitions in a unimolecular (two-state) DNA origami switch and in a bimolecular DNA origami reaction system. SAXS can monitor the conformational transitions of macromolecules and their assemblies in free solution under virtually arbitrary solution conditions. ${ }^{20,23-27}$ SAXS does not require any labeling and directly probes the global conformation in solution. By using a stopped-flow kinetic mixer to trigger conformational 
changes by rapid changes in $\mathrm{MgCl}_{2}$ concentration and a high-flux synchrotron beamline for fast data acquisition, ${ }^{28}$ we achieved a time resolution of $25 \mathrm{~ms}$ (Fig. 1a). We applied trSAXS to study the dynamics of a DNA origami switch (switchD16) ${ }^{19}$. We have previously characterized the structure and equilibrium conformations of switchD16 ${ }^{19,20}$. In brief, switchD16 consists of two $\sim 100 \mathrm{~nm}$ long rigid arms connected by a Holliday junction at the center that allows for reversible transitions between an open and a closed conformation (Fig. 1). Shape-complementary patterns of blunt-ended double helical protrusions and recessions are arranged on both arms that can form 16 basepair stacking interactions in the closed conformation of the switch. Electrostatic repulsion counteracts the stacking contacts, such that at low cation concentration the open conformation is favored. We have previously demonstrated that SAXS can monitor and quantify the $\mathrm{MgCl}_{2}$-dependent unimolecular equilibrium of the switchD16 device ${ }^{20}$ and determined the midpoint of the open-to-close transition at $\sim 10 \mathrm{mM} \mathrm{MgCl} 2$ (Fig. 1b and Supporting Information). In the trSAXS measurements, we started with DNA switch devices in $5 \mathrm{mM} \mathrm{MgCl}_{2}$ where the open conformation is predominantly populated (fraction closed $f_{\text {closed }}<4 \%{ }^{20}$. Using the stoppedflow mixer (see Supporting Information), we then rapidly (within $\leq 1 \mathrm{~ms}$ ) added $\mathrm{MgCl}_{2}$ to final concentrations of 15,25 , and $35 \mathrm{mM}$ (where in equilibrium $f_{\text {closed }}=90 \%, 98 \%$, and $99 \%$, respectively) and monitored the subsequent conformational changes. For the $15 \mathrm{mM} \mathrm{MgCl} 2$ condition the transition from the open to the closed state is resolved and well described by a first-order kinetic model (Fig. 2 and Supporting Fig. S2) with a closing rate constant $k_{\text {close }}=$ $22 \mathrm{~s}^{-1}$ and an opening rate constant $k_{\text {open }}=2.4 \mathrm{~s}^{-1}$. For the 25 and $35 \mathrm{mM} \mathrm{MgCl} \mathrm{M}_{2}$ conditions, the transition to the closed state occurs essentially within the dead time of our instrument (25 $\mathrm{ms})$. Analysis of the data suggests a lower limit on the closing rate constants of $k_{\text {close }}=150 \mathrm{~s}^{-1}$ (Fig. 2b and Supporting Fig. S3). The measured closing times of $\tau_{\text {close }}=k_{\text {close }}{ }^{-1} \leq 7 \mathrm{~ms}$ are at most 1-2 orders of magnitude slower than a simple estimate of the time scale for the diffusive motion of the two arms from the open into the closed state of $\tau_{\text {diff }} \sim 100 \mu$ s (Supporting Text and Supporting Fig. S4), suggesting that the DNA switch closes near the diffusive speed limit. Residual energy barriers, if any, for closing must be lower than $\ln \left(\tau_{\text {close }} / \tau_{\text {diff }}\right) \sim 4 k_{B} T$, where $k_{B} T$ is the thermal energy.

\section{Slow dimerization of DNA origami bricks}

As a reference construct, we studied the kinetics of a bimolecular reaction system consisting of two separate monomeric DNA origami bricks that dimerize via shape-complementary basepair stacking interactions, similar to the switch device (Fig. 3a). The SAXS profiles at 20 
$\mathrm{mM} \mathrm{MgCl} 2$ show pronounced differences between the monomer and dimer scattering patterns (Fig. 3b). In particular, the scattering profile of the dimeric complex exhibits a clear peak at $q$ $\approx 0.28 \mathrm{~nm}^{-1}$ (Fig. 3b), which corresponds to a length scale of $2 \pi / q \approx 23 \mathrm{~nm}$ that matches the cross-section of the dimer (22 nm x $24 \mathrm{~nm}$ ) (Fig. 3a). This strong interference peak is largely missing in the monomeric profile due to the asymmetric cross sectional area (11 nm x $24 \mathrm{~nm})$ (Fig. 3a). Based on a two-state model we determined the fraction of dimers at each time point (Fig. 3c,d, Supporting Fig. S5 and Supporting Information). As expected for a bimolecular system, we find concentration dependent assembly kinetics. From a fit of a bimolecular reaction kinetics model to the data we obtain a negligible dissociation rate constant $k_{\text {off }}$ and an estimate of an association rate constant $k_{\text {on }}$ of $1.7 \cdot 10^{4} \mathrm{M}^{-1} \cdot \mathrm{s}^{-1}$. A simple estimate for the time scale of diffusive reaction that takes into account the global dimensions of the bricks yields a diffusion-limited reaction rate constant $k_{\text {diff }}$ of $\sim 2.5 \cdot 10^{8} \mathrm{M}^{-1} \cdot \mathrm{s}^{-1}$ (see Supporting Information). Hence, the brick system reacts much slower than the diffusive speed limit, which points to the existence of additional barriers, likely to overcome electrostatic repulsion and to achieve the correct alignment of the brick monomers ${ }^{29}$, that slow down the reaction.

Taken together, these data suggest that the very rapid closing transition of the DNA switch device is due to the fact that the central Holliday junction links the two arms and orients them favourably to form the 16 basepair stacking interactions that stabilize the closed state. Therefore, the central pivot link creates a high effective concentration (estimated to be $k_{\text {close }} /$ $k_{\text {on }} \sim 1.4 \mathrm{mM}$ ) of the two arms enabling them to interact on the millisecond timescale.

In conclusion, we have demonstrated that $\sim 5$ MDa DNA origami devices can undergo very fast conformational dynamics on the millisecond timescale, suggesting that such devices could be employed for switching and sensing molecular capabilities with rapid response times. While the kinetics for the DNA switch device are very fast and approach the estimated diffusion controlled rate, assembly of the DNA bricks at $n \mathrm{M}-\mu \mathrm{M}$ concentrations is much slower than both the dynamics of the DNA switch, the estimated diffusion controlled rate, and the maximal rate for toehold-mediated strand exchange. In particular since hierarchical assembly of (various types of) DNA bricks forms the basis for creating higher-order DNA structures reaching the $\sim$ GDa-range ${ }^{8,10}$, it is desirable to better understand -and ultimately optimize- their assembly kinetics. Unforeseen barriers that could slow down the dimerization dynamics could be caused by deviations of the actual brick geometry from the designed one. For example, residual twist in honeycomb structures ${ }^{8}$ may make it necessary that the bricks deform through thermal fluctuations in order to satisfy the stacking bonds. The associated 
energetic penalties will be likely on the order of multiple $k_{B} T$ and could thus explain the slow binding rates that we have observed. Thus, one approach for increasing the speed of DNA brick association may consist in more precise design. Other approaches might include optimized temperature and salt conditions, introduction of confinement or molecular crowding agents, chemical modifications to the DNA to reduce charge repulsion, and a more symmetric design to facilitate correct alignment.

Our work establishes trSAXS as a powerful tool to monitor large-scale conformational changes and assembly of DNA origami objects on timescales from milliseconds to hours without the need for labeling or surface immobilization. The ability of SAXS to directly probe the global conformation in solution is advantageous, since it has recently been demonstrated that inferring global conformations from local distance measurements e.g. by Förster resonance energy transfer (FRET) can be problematic ${ }^{30,31}$ and that the addition of dyes can bias the conformational ensemble ${ }^{32}$. In the future, a combination of continuous-flow mixing in appropriate microfluidics with microfocus X-ray sources has the potential to push the timeresolution even into the $\mu$ s-regime, ${ }^{33}$ which would allow us to directly probe conformational transitions at the molecular speed limit.

\section{ASSOCIATED CONTENT}

Supporting Methods, Supporting Text, and Supporting Figures S1-S8. This material is available free of charge via the Internet at http://pubs.acs.org.

\section{AUTHOR INFORMATION}

\section{Corresponding author}

*E-mail: Jan.Lipfert@1mu.de. Phone: +49-89-2180-2005

Author contributions. All authors designed this study. T.G. assembled and purified DNA origami samples; L.K.B. and P.W. performed SAXS measurements and analyzed the data. All authors contributed to writing the paper and have given approval to the final version of the manuscript.

\section{Funding}

This work was supported by the Deutsche Forschungsgemeinschaft through grants provided within Gottfried-Wilhelm Leibniz Program, the Excellence Clusters CIPSM (Center for Integrated Protein Science Munich), NIM (Nanosystems Initiative Munich), and the Sonderforschungsbereich SFB863. 


\section{ACKNOWLEDGEMENTS}

We thank Dr. Theyencheri Narayanan for assistance at beam line ID02 and Dr. Martha Brennich for help at beam line BM29 at the ESRF (Grenoble, France), Dr. Martin Schroer for assistance at beam line P12 at DESY (Hamburg, Germany), Florian Praetorius for scaffold DNA preparations, and Philip Tinnefeld for discussions. 


\section{FIGURES}

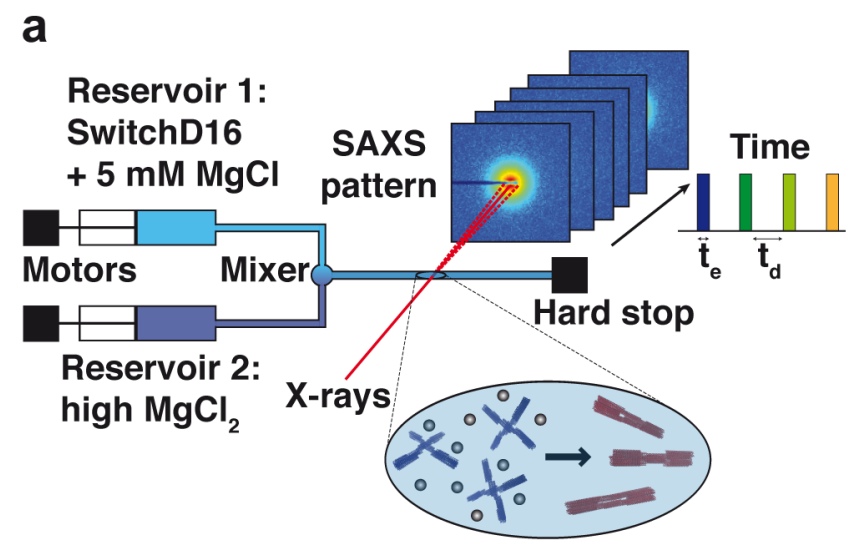

SwitchD16

b
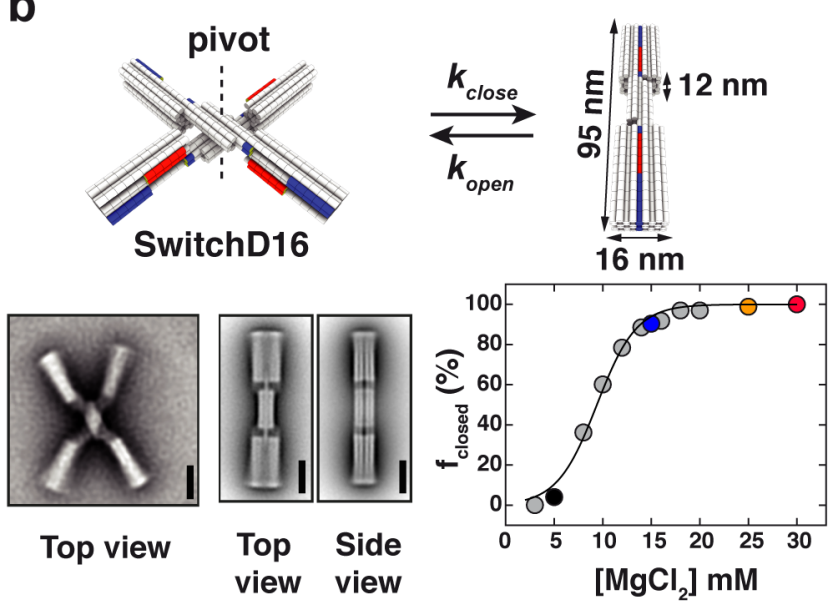

Figure 1. Schematic of time-resolved SAXS and a $\mathbf{M g C l}_{2}$-dependent DNA origami switch device. (a) Schematic of the stopped-flow mixing device coupled to the high brilliance beamline ID02 at the ESRF. The mixing reservoirs 1 and 2 contain the switchD16 device in $5 \mathrm{mM} \mathrm{MgCl} 2$ and buffer with high $\mathrm{MgCl}_{2}$ concentrations, respectively. After turbulent mixing, the mixture is directed to a capillary and the flow is stopped. The beam hits the sample in a specific acquisition pattern defined by the exposure time $\left(t_{e}\right)$ and a delay time $\left(t_{d}\right)$ (see Supporting Information). (b) (Top) Schematic view of the switchD16 device, which changes from an open to a closed conformation upon the addition of $\mathrm{MgCl}_{2}$. The closed state is stabilized by 16 basepair stacking interactions occurring at the interface of shapecomplementary double helical protrusions (red) and recessions (blue). (Bottom, left) Negative-stain TEM micrographs of switchD16 particles in the presence of $5 \mathrm{mM}$ and $25 \mathrm{mM}$ $\mathrm{MgCl}_{2}$. (Scale bars, $20 \mathrm{~nm}$ ). (Bottom, right) Equilibrium fraction of closed switchD16 devices as a function of $\mathrm{MgCl}_{2}$ determined by SAXS and two-state based thermodynamic model (black line). 

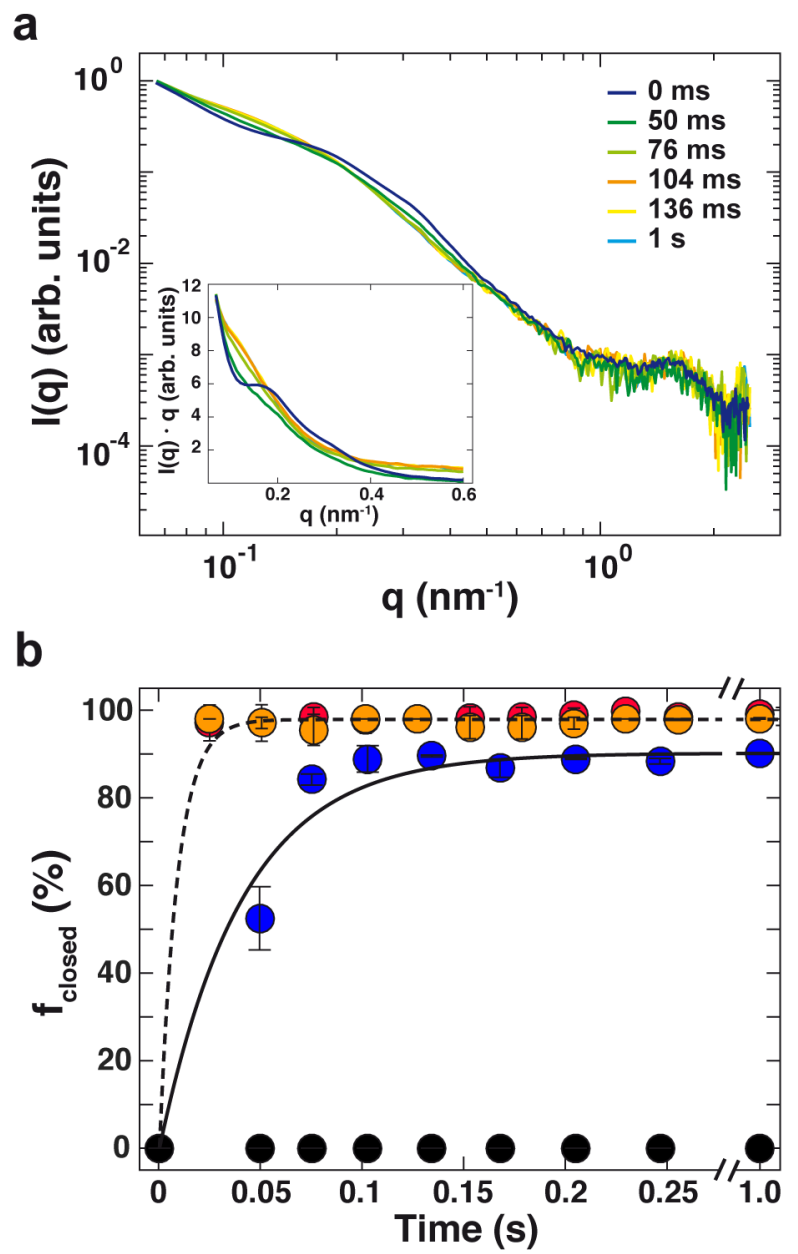

Figure 2. TrSAXS reveals conformational kinetics of a DNA origami switch device. (a) SAXS profiles for switchD16 for selected time points after increasing $\mathrm{MgCl}_{2}$ concentration to $15 \mathrm{mM}$. The inset shows a zoom of the data in Holzer representation $(I(q) \cdot q$ vs. $q$ ). (b) Fraction of switchD16 devices in the closed conformation vs. time after changing to $\mathrm{MgCl}_{2}$ concentrations of: $5 \mathrm{mM}$ (black circles), $15 \mathrm{mM}$ (blue circles), $25 \mathrm{mM}$ (orange circles) and $35 \mathrm{mM}$ (red circles). Symbols and error bars are the mean and standard deviation from two independent repeats of each condition. The solid black line and dashed black line represent a reversible unimolecular first-order reaction fit to the data at $15 \mathrm{mM}$ and $25 \mathrm{mM}$ $\mathrm{MgCl}_{2}$ (see Supporting Information). 
a

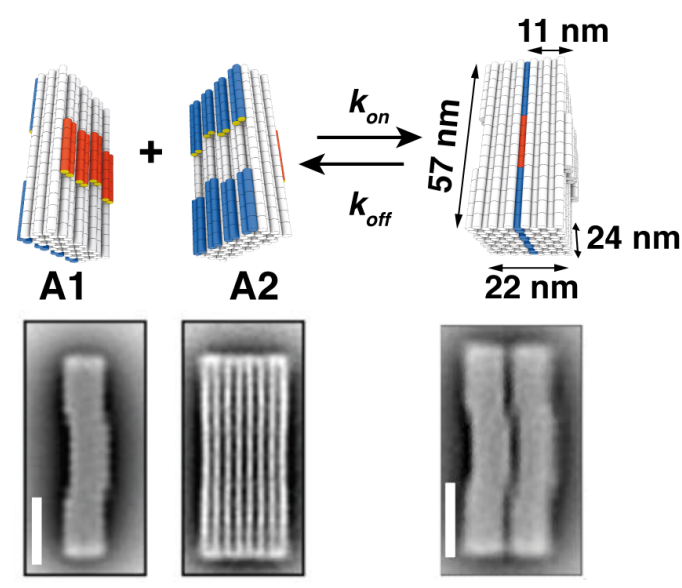

C

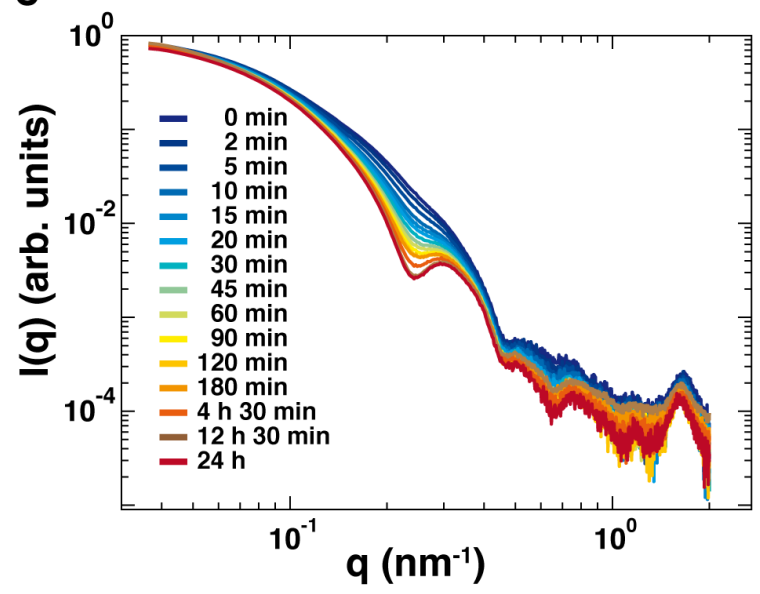

b

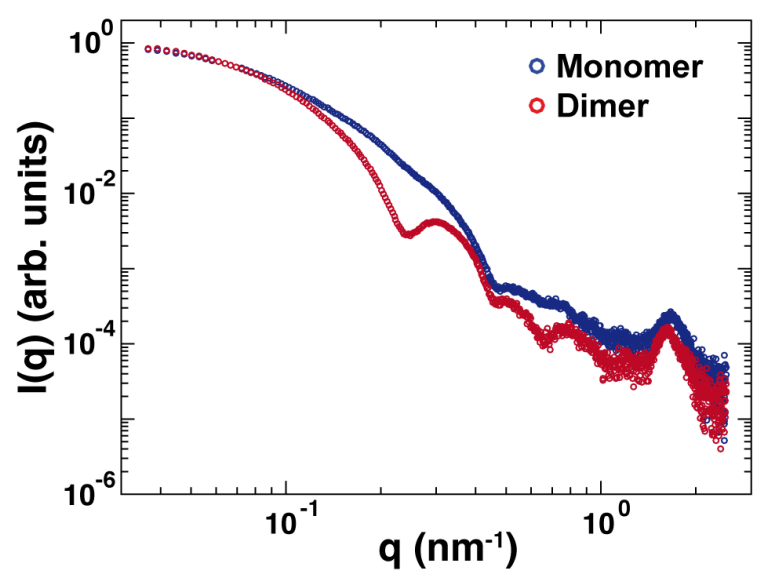

d

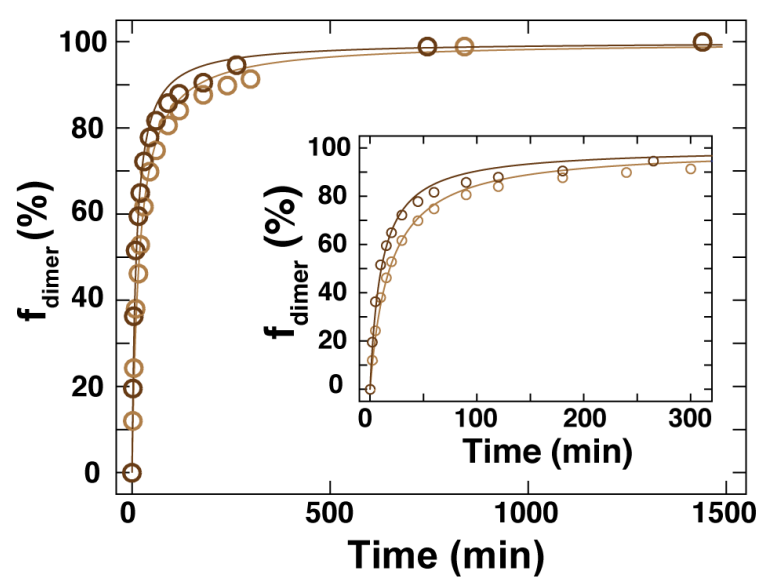

Figure 3. Time-resolved SAXS measurements on dimerization kinetics of DNA origami bricks. (a) (Top) Schematic of DNA origami brick monomers with double helical protrusions and recessions (indicated in red and blue) allowing for the formation of a dimeric brick in the presence of $20 \mathrm{mM} \mathrm{MgCl}_{2}$. (Bottom) TEM micrographs of DNA origami monomers and dimers. Scale bar: $20 \mathrm{~nm}$. (b) Experimental scattering profiles of DNA origami monomers (blue circles) and dimers (red circles) at a sample concentration of $100 \mathrm{nM}$ in $20 \mathrm{mM} \mathrm{MgCl}$. (c) Time evolution of scattering profiles after 1:1 mixing of monomeric brick samples at an initial concentration of $100 \mathrm{nM}$. (d) Fraction of dimeric brick particles as a function of time determined from a two-state model for initial monomer concentrations of $100 \mathrm{nM}$ (dark brown circles) and $50 \mathrm{nM}$ (light brown circles). Solid lines represent fits of an irreversible bimolecular reaction rate model (see Equation 9 Supporting Information) yielding an average association reaction rate constant of $1.7 \cdot 10^{4} \mathrm{M}^{-1} \cdot \mathrm{s}^{-1}$. The inset shows a close up of the early time points. 


\section{REFERENCES}

(1) Siavashpouri, M.; Wachauf, C. H.; Zakhary, M. J.; Praetorius, F.; Dietz, H.; Dogic, Z. Nat. Mater. 2017, 16, 849-856.

(2) Halley, P. D.; Lucas, C. R.; McWilliams, E. M.; Webber, M. J.; Patton, R. A.; Kural, C.; Lucas, D. M.; Byrd, J. C.; Castro, C. E. Small 2016, 12, 308-20.

(3) Schmied, J. J.; Gietl, A.; Holzmeister, P.; Forthmann, C.; Steinhauer, C.; Dammeyer, T.; Tinnefeld, P. Nat. Methods 2012, 9, 1133-4.

(4) Ponnuswamy, N.; Bastings, M. M. C.; Nathwani, B.; Ryu, J. H.; Chou, L. Y. T.; Vinther, M.; Li, W. A.; Anastassacos, F. M.; Mooney, D. J.; Shih, W. M. Nat. Commun. 2017, 8, 15654.

(5) Rothemund, P. W. Nature 2006, 440, 297-302.

(6) Douglas, S. M.; Dietz, H.; Liedl, T.; Hogberg, B.; Graf, F.; Shih, W. M. Nature 2009, 459, 414-8.

(7) Dietz, H.; Douglas, S. M.; Shih, W. M. Science 2009, 325, 725-30.

(8) Wagenbauer, K. F.; Sigl, C.; Dietz, H. Nature 2017, 552, 78-83.

(9) Tikhomirov, G.; Petersen, P.; Qian, L. Nature 2017, 552, 67.

(10) Ong, L. L.; Hanikel, N.; Yaghi, O. K.; Grun, C.; Strauss, M. T.; Bron, P.; Lai-KeeHim, J.; Schueder, F.; Wang, B.; Wang, P.; Kishi, J. Y.; Myhrvold, C.; Zhu, A.; Jungmann, R.; Bellot, G.; Ke, Y.; Yin, P. Nature 2017, 552, 72.

(11) Marras, A. E.; Zhou, L.; Su, H. J.; Castro, C. E. Proc. Natl. Acad. Sci. U. S. A. 2015, $112,713-8$.

(12) Zhang, D. Y.; Winfree, E. J. Am. Chem. Soc. 2009, 131, 17303-17314.

(13) Andersen, E. S.; Dong, M.; Nielsen, M. M.; Jahn, K.; Subramani, R.; Mamdouh, W.; Golas, M. M.; Sander, B.; Stark, H.; Oliveira, C. L.; Pedersen, J. S.; Birkedal, V.; Besenbacher, F.; Gothelf, K. V.; Kjems, J. Nature 2009, 459, 73-6.

(14) Simmel, F. C.; Yurke, B. Appl. Phys. Lett. 2002, 80, 883-885.

(15) Pan, J.; Li, F.; Cha, T. G.; Chen, H.; Choi, J. H. Curr. Opin. Biotechnol. 2015, 34, 5664.

(16) Zhang, D. Y.; Seelig, G. Nat. Chem. 2011, 3, 103-13.

(17) Rajendran, A.; Endo, M.; Hidaka, K.; Sugiyama, H. J. Am. Chem. Soc. 2013, 135, 1117-23.

(18) Kuzyk, A.; Yang, Y.; Duan, X.; Stoll, S.; Govorov, A. O.; Sugiyama, H.; Endo, M.; Liu, N. Nat. Commun. 2016, 7, 10591.

(19) Gerling, T.; Wagenbauer, K. F.; Neuner, A. M.; Dietz, H. Science 2015, 347, 1446-52.

(20) Bruetzel, L. K.; Gerling, T.; Sedlak, S. M.; Walker, P. U.; Zheng, W.; Dietz, H.; Lipfert, J. Nano Lett. 2016, 16, 4871-9.

(21) Russell, R.; Zhuang, X.; Babcock, H. P.; Millett, I. S.; Doniach, S.; Chu, S.; Herschlag, D. Proc. Natl. Acad. Sci. U. S. A. 2002, 99, 155-60.

(22) Chen, S. J.; Dill, K. A. Proc. Natl. Acad. Sci. U. S. A. 2000, 97, 646-51.

(23) Lipfert, J.; Doniach, S. Annu. Rev. Biophys. Biomol. Struct. 2007, 36, 307-27.

(24) Svergun, D., I. ; Koch, M. H. J. Rep. Prog. Phys. 2003, 66, 1735.

(25) Pollack, L.; Doniach, S., Time-resolved X-ray scattering and RNA folding. In Methods in Enzymology: Biophysical, Chemical, and Functional Probes of RNA Structure, Interactions and Folding: Part B, Elsevier Inc.: 2009; Vol. 469, pp 253-268.

(26) Hura, G. L.; Menon, A. L.; Hammel, M.; Rambo, R. P.; Poole, F. L., 2nd; Tsutakawa, S. E.; Jenney, F. E., Jr.; Classen, S.; Frankel, K. A.; Hopkins, R. C.; Yang, S. J.; Scott, J. W.; Dillard, B. D.; Adams, M. W.; Tainer, J. A. Nat. Methods 2009, 6, 606-12.

(27) Fischer, S.; Hartl, C.; Frank, K.; Rädler, J. O.; Liedl, T.; Nickel, B. Nano Lett. 2016, 16, 4282-4287.

(28) Panine, P.; Finet, S.; Weiss, T. M.; Narayanan, T. Adv. Colloid Interface Sci. 2006, $127,9-18$. 
(29) Kilchherr, F.; Wachauf, C.; Pelz, B.; Rief, M.; Zacharias, M.; Dietz, H. Science 2016, 353.

(30) Song, J.; Gomes, G.-N.; Shi, T.; Gradinaru, C. C.; Chan, H. S. Biophys. J. 2017, 113, 1012-1024.

(31) Fuertes, G.; Banterle, N.; Ruff, K. M.; Chowdhury, A.; Mercadante, D.; Koehler, C.; Kachala, M.; Estrada Girona, G.; Milles, S.; Mishra, A.; Onck, P. R.; Grater, F.; EstebanMartin, S.; Pappu, R. V.; Svergun, D. I.; Lemke, E. A. Proc. Natl. Acad. Sci. U. S. A. 2017, 114, E6342-e6351.

(32) Kugel, W.; Muschielok, A.; Michaelis, J. Chemphyschem 2012, 13, 1013-22.

(33) Graceffa, R.; Nobrega, R. P.; Barrea, R. A.; Kathuria, S. V.; Chakravarthy, S.; Bilsel, O.; Irving, T. C. J. Synchrotron Radiat. 2013, 20, 820-5. 
For TOC only

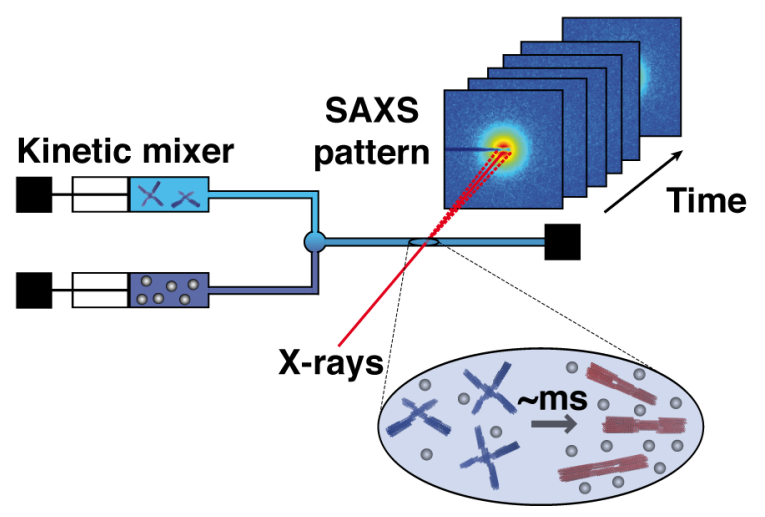

